\title{
The impact of oral health on quality of life: questionnaires most commonly used in the literature
}

\begin{abstract}
The aim of this study is to identify the main questionnaires that assess the impact of oral health conditions on quality of life, and their characteristics. This study was constructed using the descriptors "Oral Health", "Sickness Impact Profile", "Quality of Life", "Questionnaires", "Cost of Illness" and "Health Evaluation", in Portuguese and English, between 1997 and 2013, from PubMed. Among the main questionnaires are the OIDP, the GOHAI, and the OHIP-14, a shortened version of the OHIP-49. The OIDP is a sociodental indicator that, by assessing the frequency and severity of impacts that affect the performance of individuals, provides a score of individual impact, making it more consistent for use in assessing treatment needs. It was observed that the OHIP-14 is the most widely used instrument to assess the adverse impact caused by oral conditions on well-being and quality of life. Its advantages are the possibility of the researcher explaining the research objectives, guiding the administration of the instrument, and more uniform and useful data. While GOHAI is an instrument used for assessment, versus measuring the state of oral health of the patient. Thus, GOHAI is more sensitive in detecting impacts such as dysfunction and pain, while the OHIP-14 is better at detecting the psychological impacts. Thus, one should recognize the importance of the impact of oral health conditions on the quality of life of individuals, taking into account social and psychosocial dimensions.
\end{abstract}

Keywords: oral health, sickness impact profile, quality of life, questionnaires, cost of illness, health evaluation
Volume 8 Issue 5 - 2017

\section{Pamella Valente Palma,' Paula Liparini Caetano,' Isabel Cristina Goncalves Leite ${ }^{2}$ 'School of Dentistry, Federal University of Juiz de Fora, Brazil ${ }^{2}$ Department of Public Health, Federal University of Juiz de Fora, Brazil}

Correspondence: Isabel Cristina Goncalves Leite, Department of Public Health, Federal University of Juiz de Fora, rua Jose Lourenco Kelmer, Campus Universitário-São Pedro, 36036-900 Juiz de Fora, Minas Gerais, Brazil, Email isabel.leite@ufjf.edu.br

Received: October 18,2017 | Published: November 01, 2017

\section{Introduction}

Quality of life is related to a number of elements such as selfesteem, personal well-being, functional ability, socioeconomic status, emotional state, social interaction, intellectual activity, selfcare, family support, one's proper state of health, cultural values, ethics, and religiousness, lifestyle, satisfaction with one's job and / or daily activities, and environment in which one lives. Thus, in populations with chronic diseases, various quality of life indices are frequently used in order to determine the impact of health care, especially when no cure exists. Meanwhile, there are some oral conditions that negatively affect quality of life, such as tooth loss, decay, malocclusions, fluorosis, and the use of prosthetics. ${ }^{1}$ There is a direct relationship between social impact and perceived need for dental care by the patient. Thus, for the planning of dental services, self-perceived oral health condition is used as tool. ${ }^{2}$ Admittedly, the use of quality of life indicators has become essential in dental research and clinical studies, especially those that evaluate prevention and seek therapeutic modalities to improve the individual's state of health. These instruments provide different information depending on age, since the cognitive ability for understanding and self-perception changes when comparing a child with an elderly person, for example. Through them, and with the use of numerical scores, you can compare groups with or without disease in the oral cavity, in pre- and posttreatment stages, with different diseases, or with different degrees of severity of the same disease. This will play a role in determining the extent of change, in well-being, and in the quality of life of patients, which will help in the treatment. ${ }^{3}$ The aim of this study was to review in the literature some instruments that assess quality of life related to oral health, describing those most commonly used, and allowing clinicians to access and understand their influence on the process approaching and treating their patients. The review was made with the use of the descriptors "Oral Health", "Sickness Impact Profile", "Quality of Life", "Questionnaires", "Cost of Illness", and "Health Evaluation", in Portuguese and English, over the period from 1997 to 2013, from PubMed.

\section{Review}

There are various quality of life indices, general or specific, developed to assess the impact of oral diseases on quality of life. The main ones are: Dental Impact on Daily Living (DIDL), Oral Health Impact Profile (OHIP), World Health Organization Quality of Life (WHOQOL), Oral Health Impact Profile - short version (OHIP-14), United Kingdom Oral Health-Related Quality of Life (OHQoL-UK), Oral Impacts on Daily Performance (OIDP), EuroQoL (EQ), and other indices.

\section{World Health Organization Quality of Life (WHOQOL)}

After noting the lack of a quality of life assessment instrument with a cross-cultural focus, the WHO developed a unique methodology for its creation. Initially, the World Health Organization Quality of Life (WHOQOL-100) was developed, an instrument composed of 100 questions. The need for a quicker instrument for use in extensive epidemiological studies led the WHO to develop a shortened version with 26 questions (the WHOQOL-BREF). Two modules are currently being developed: one specifically to assess quality of life in patients 
with HIV/AIDS, and another to evaluate spirituality, religiousness, and personal beliefs. ${ }^{4}$

\section{EuroQoL}

This is an instrument developed by the EuroQol group (Group of European Multidisciplinary Researchers) translated into 5 principal languages: Dutch, English (UK), Finnish, Norwegian, and Swedish, and recently into other languages as well. It is characterized by being self-administered and short enough to be used in conjunction with other measurements. It consists of two main components. The first is a descriptive system that defines quality of life relative to health in five dimensions (mobility, self-care, normal activities, pain/discomfort, and anxiety/depression), each with three levels of severity (1-no problems / 2-some problems / 3-extreme problems). It classifies respondents into one of 243 distinct health states, with a five-digit code. The Total EuroQol Index, which corresponds to the "true" state of health, is based on a formula where each dimension has a different weight in the assessment of health. The second component consists of the Visual Analogue Scale (VAS) system, a numbered scale from 0 to 100 , where 0 is the worst imaginable health status and 100 the best Its disadvantages are that, when compared with other measurements, it has more missing data and was designed to be used only in face to face interviews. ${ }^{5}$

\section{Dental Impact on Daily Living (DIDL)}

The DIDL is an instrument to measure the perception of oral health and quality of life, developed and adapted to the values of the Brazilian culture. It consists of a questionnaire consisting of 36 items to assess various impacts in five dimensions and a Likert-type scale, a method developed to score the value assigned to each dimension by the participant. The dimensions are given weights proportional to the impact perceived by the subject, using a graduated visual scale from 1 to 10 , with the dimensions placed side by side. ${ }^{6}$ It assesses psychosocial problems reflected in quality of life according to the individual's oral condition, using five quality-of-life dimensions: comfort (related to gingival health and absence of food impaction), appearance (individual's self-image), pain, performance (ability to carry out normal daily activities and interact socially), and dietary restrictions (in biting and chewing). ${ }^{7}$ The main advantages of this index are its flexibility in producing or eliminating data (individual items, dimensions, or the total score) and the possibility of assigning weights to the dimensions, reflecting the real importance of each dimension in the life of the individual. ${ }^{3}$

\section{Oral Health Impact Profile (OHIP)}

This is the instrument most commonly used for assessing the adverse impact caused by oral conditions on well-being and quality of life. It is better at detecting psychological impacts and its biggest advantage is that the questions were derived from a representative group of patients and not prepared by researchers. ${ }^{8}$ It was developed and tested by Slade and Spencer (1994), originally with 49 questions, and later its reduced version was formulated with 14 questions, keeping the same dimensions. The OHIP works with 7 dimensions of impact: functional limitation, physical pain, psychological discomfort, physical incapacity, psychological incapacity, social incapacity, and inability to perform daily activities. ${ }^{9}$ The OHIP was initially prepared in English, in a different socio-cultural context, and has been translated into more than 15 languages, including Portuguese. ${ }^{10}$ Currently, the OHIP-14 questionnaire has become a method of choice for evaluations that need to capture perceptions and feelings of individuals about their own oral health and their expectations regarding dental treatment and services. ${ }^{11}$ The OHIP is a subjective indicator that aims to provide a comprehensive measure of dysfunction, discomfort, and self-assessed incapacity attributed to oral conditions, providing information on the perceived impacts produced by oral problems and the effectiveness of health services in mitigating these impacts. ${ }^{12}$

\section{United Kingdom Oral Health-Related Quality of Life (OHQoL-UK)}

This instrument was developed using opinions from the UK population to identify the principal areas of oral health-related quality of life. The index on a local study in London, demonstrated good psychometric properties in terms of validity and reliability. The simple unweighted version, (OHQoL-UK) asks participants to answer "What effect do your teeth, gums, mouth and / or bridgework have on each of the 16 key areas?" With options like "very bad" (score 1), "bad" (score 2), "none" (score 3), "good" (score 4) or "very good" (score 5). By totaling the responses from each of the 16 items, an overall score ranging from 16 to 80 can result. This simple unweighted version of the measure was used because the quality of life instrument related to the oral health of the UK does not appear to improve its psychometric properties. ${ }^{13}$

\section{Oral Impacts on Daily Performance (OIDP)}

The OIDP is a socio-dental indicator that, by assessing the frequency and severity of impacts that affect the daily performance of individuals, provides an individual impact score. Oral problems and symptoms perceived by the subjects as having impact are investigated in order to relate this to the clinical condition, which makes the OIDP more coherent for use in assessing treatment needs. ${ }^{14}$

\section{Geriatric Oral Health Assessment Index (GOHAI)}

The GOHAI was originally developed for elderly populations, but is also indicated for adults of all ages. ${ }^{15}$ The GOHAI has been used in populations of young adults. ${ }^{16}$ and is an instrument used for assessment, and is not intended for measuring the oral health status of the patient. ${ }^{17}$ According to Dolan, ${ }^{18}$ for the purposes of developing the GOHAI, oral health was defined as the absence of pain and infection, consisting of a comfortable and functional dentition (natural or prosthetic), yet allowing the individual to carry on their social lives. ${ }^{18}$

The GOHAI consists of twelve items that are related to three basic functions:

a. Physical, including eating, speaking, and swallowing.

b. Psychological, including concern or care for one's own oral health, dissatisfaction with appearance, self-consciousness regarding oral health, and the avoidance of social contacts due to dental problems.

c. Pain or discomfort, considering the use of drugs to relieve these feelings, provided they come from the mouth. ${ }^{19}$

\section{Discussion}

Quality of life may be assessed and quantified through psychometric properties such that the data are reproducible and influenced by age and by time. These properties refer to reliability, validity, 
responsiveness, sensitivity, and specificity. Reliability is the accuracy of the measurement (internal consistency, retest); validity, the correct representation of the concept to be measured in the questionnaire; responsiveness, perception of recognized changes in clinical status; sensitivity, the proportion of true positive individuals among patients; and specificity, proportion of true negative individuals among nonpatients. ${ }^{20}$ The instruments used to assess quality of life related to health can be differentiated by various elements such as the scope (generic, for patients with chronic diseases, or for specific conditions), the age of the individuals who will respond to it, form of administration, response mode, purpose of evaluation, or by the development method. ${ }^{21}$ The WHOQOL-100 consists of one hundred questions regarding the six domains: physical, psychological, level of independence, social relationships, environment, and spirituality/ religiousness/personal beliefs. The need for a shorter instrument that would take little time to complete, and would retain satisfactory psychometric characteristics, led the WHO Quality of Life Group to develop an abbreviated version of the WHOQOL-100, the WHOQOL-BREF. The abridged version presents 26questions. ${ }^{4}$ The WHOQOL was developed assuming that the concept of quality of life is comprehensive and can be applied to various diseases and to nonmedical situations. However, for some groups of people or diseases, the WHOQOL may not assess quality. The first module of the WHOQOL to be developed was for HIV/AIDS, due to the medical importance of the disease, the impact on quality of life, its stigma, and the peculiarities involving the contagion. ${ }^{4-21}$ The deepening of the spirituality, religiousness, and personal beliefs domain, in different cultures and religions, the facets that comprise them, underlie the creation of a new WHOQOL. Thus, it may be possible and interesting to develop a module from the already existing measure in the WHOQOL and make it a more sensitive and complete measure of this domain for use in the area of health. In this case, it would be introduced as an additional module to the WHOQOL, to be used when necessary. It is important to note that this module is not directed at any specific religion, but at all forms of spirituality, practiced or not through formal religions. For those who are not affiliated with any religion or spiritual dimension, the domain should refer to beliefs or codes of behavior. ${ }^{22}$. The creation of instruments to assess quality of life is a much more complex task than developing instruments and forms of assessment of mortality and morbidity. ${ }^{4}$ The understanding of quality of life is related to a great many fields of knowledge that are continuously interconnected to the human, biological, social, political, economic, cultural, medical, among others, making the planet increasingly connected. ${ }^{23}$ The most frequently used instruments to measure quality of life among the adult and elderly population are the OHIP-14, followed by the GOHAI, the latter having been initially developed for the elderly. ${ }^{24}$ The GOHAI was created in 1990, and is an index of perceived oral health impact on the daily lives of the elderly. In this index, oral health is understood as the absence of pain and infection, consisting of a comfortable and functional dentition (natural or prosthetic) which enables the individual to perform his/her role in society. ${ }^{25}$ Being an assessment instrument and not an objective measure of oral health, the GOHAI cannot be used to diagnose oral diseases. However, at the individual level, its use by other professionals such as a geriatrician may indicate the need for a clinical oral examination, and at the populational level, it would be a means of gathering information about oral problems, or even a form of evaluating dental treatment outcomes. ${ }^{17}$ The OHIP-14 is a subjective indicator that was developed with the goal of providing a comprehensive measure of dysfunction, discomfort, and incapacity attributed to one's self-perceived and self-assessed oral condition. ${ }^{26}$ The possibility of gathering information on the perception of impacts produced by oral problems and the effectiveness of health services in mitigating these impacts through the OHIP-14 is one of its major uses. ${ }^{12-27}$ A study was conducted in 2008 by Chapelin, Barcellos and Miotto, in the city of Viana (ES) with 98 patients who agreed to participate in a standardized interview, on the following topics: sociodemographic situation, dental practice structure, and the OHIP-14. Only 53 patients had completed treatment, and they answered the OHIP again. The results indicated a greater impact on the psychological discomfort and physical pain dimensions. On the other hand, $78 \%$ of the patients did not perceive the impact of oral health on their quality of life. While the individuals over 40 years old had a 2.6 times greater chance of suffering impact from oral health problems on their quality of life. Of the patients who began treatment, with impacts, it can be stated that dental treatment improved the quality of life of these patients, since $77.8 \%$ had reduced impact after its completion. ${ }^{12}$ The development of subjective indicators based on the individual perception of the health/illness process was stimulated by the need for subjective awareness of one's health condition or the impact of oral health on quality of life..$^{27}$ There is a direct relationship between social impact and perceived need for dental care by the patient. Thus, selfperception of one's oral health condition can be used as a tool in the planning of dental services. The OHIP proved to be a useful instrument for screening priority groups for access to the service. ${ }^{2-29}$ In comparative studies, the OHIP-14 and the GOHAI present a strong correlation. ${ }^{24}$ where the first is better for detecting psychological impacts, while the second is better at detecting impacts regarding dysfunction and pain. ${ }^{30-32}$ With the dissemination of subjective indicators, highlighting the creation of OHIP in 1994, Dental Impact on Daily Living (DIDL) was created, an instrument to estimate different impacts in five dimensions. In general, it simply combines gender, socioeconomic status, and clinical variables as possible predictors of impact. ${ }^{33}$ The instrument was tested on a convenience sample of Brazilians, in which its stability (test-retest) and internal consistency were evaluated as yielding good results, for the questionnaire ( 0.87 and 0.85 , respectively), and for the scale ( 0.78 and 0.59 , respectively). ${ }^{3}$ The OIDP assesses the impacts of oral conditions on the capacity of individuals to perform eight daily activities, such as: eating, speaking, personal hygiene, ability to perform occupational activities, social relations, smiling, and emotional state and sleep. This indicator has adequate psychometric properties and a consistent theoretical basis in different populations, proving to be reliable and valid in cross-sectional population studies. Internal consistency demonstrated high reliability (Cronbach's alpha $=0.65$ ), and the testretest demonstrated the stability of the index, conducted with 47 individuals over a 3 week interval, obtaining kappa coefficients from 0.95 to $1.0 .^{34}$ The OHQoL-UK instrument relates quality of life to oral health, and was developed in a local study in London, while the OHIP was developed by the WHO. ${ }^{13}$ The EuroQol is a simple instrument, short and easy to answer, and which allows comparisons between various countries. This instrument defines health states based on five dimensions (mobility, usual activities, personal care, pain/discomfort and anxiety/depression), each divided into three levels of severity (no problems, some problems, and extreme problems), totaling 243 health states. ${ }^{35}$ The evaluation of states of health on a $0-100$ scale, over the course of one year, ranging from the worst imaginable state of health to the best, by the EuroQoL questionnaire, presents good capacity of understanding by individuals, is a suitable method for describing and measuring state of health, and shows good reliability, demonstrated by 
the test-retest. ${ }^{13-35}$ The impact and effects of oral health on quality of life, including physical and social factors, can be measured by the OHQoL-UK instrument. Factors such as female gender and low income are related to a low OHQoL-UK, indicating correlation between these variables and quality of life. ${ }^{36}$

\section{Conclusion}

The importance of the impact of oral health conditions on individuals' quality of life of should be recognized, taking into account social and psychosocial dimensions. The possibility of incorporating these subjective indicators in assessing patients ought to be evaluated, as they are good self-assessment predictors of oral health.

\section{Acknowledgments}

None.

\section{Conflicts of interest}

The authors declare that there is no conflict of interest.

\section{Funding}

None.

\section{References}

1. Manuela Wanderley FLOPESI, Estela Santos G, Renato de Vasconcelos $\mathrm{A}$, et al. Impact of periodontal diseases on quality of life. RGO, Rev gaúch odontol. 2011;59(1):39-44.

2. Almeida AM, Gonçalves JR. Evaluation of perceived oral health impact using the OHIP instrument - 14 (oral health impact profile) instrumento simplified form. UFES Journal of Dentistry. 2004;6:11-16.

3. Feu D, Quintão CCA, Miguel JAM. Indicators of quality of life and their importance in orthodontics. Dental Press J Orthod. 2010;15(6):61-70.

4. Fleck MPA. World Health Organization Quality of Life Assessmen Tool (WHOQOL - 100): characteristics and perspectives. Science and Collective Health. 2006;5:33-38.

5. Brooks R. EuroQol: the current state of play. Health Policy. 1996;37(1):53-72.

6. Pandolfi M, Barcellos LA, Miotto MHMB, et al. Oral health and quality of life of users of the dental services of Vitória. Brazilian Research in Pediatric Dentistry and Integrated Clinic. 2011;3:311-316.

7. Leao A, Sheiman A. The development of a socio-dental measure of dental impacts on daily living. Community Dent Health. 1996;13(1):22-26.

8. Sanderes AE, Slade GD, Lim S, et al. Impact of oral disease on quality of life in the US and Australian populations. Community Dent Oral Epidemiol. 2009;37(2):171-181.

9. Slade GD, Spencer AJ. Development and evaluation of the Oral Health Impact Profile. Community Dent Health. 1994;11(1):3-11.

10. Oliveira BHDE, Nadanovsky P. Psychometric properties of the Brazilian version of the Oral Health Impact Profile-short. Community Dent Oral Epidemiol. 2005;33(4):307-314.

11. Trícia Drumond-Santana, Fernando Oliveira Costa, Elton Gonçalves Zenóbio, et al. Impact of periodontal disease on the quality of life of dentate diabetic individuals. Public Health Notebooks. Cadernos de Saúde Pública. 2007;23(3):637-644.

12. Chapelin CC, Barcellos LA, Miotto MHMB. Effectiveness of dental treatment and reduced impact on quality of life. UFES dentistry magazine. 2008;10:46-51.
13. Mcgrath C, Bedi R. (Population based norming of the UK oral health related quality of life measure (OHQol-UK). British Dental Journal. 193:521-524

14. Gomes AS, Abegg CO. The impact of oral health on daily performance of municipal waste disposal workers in Porto Alegre, Rio Grande do Sul State, Brazil. Cad. Saúde Pública. 2007;23(7):1707-1714.

15. Atchison KA, Der-martirosian C, Gift HC. Components of self-reported oral health and general health in racial and ethnic groups. J Public Health Dent. 1998;58(4):301-308.

16. Atchison KA. The general oral health assessment index. In: Slade GD, Editors. Measuring oral health and quality of life. Department of Dental Ecology, School of Dentistry, University of North Carolina, USA. 1997. p. $71-80$.

17. Atchison KA, Dolan TA. Development of the geriatric oral health assessment index. J Dent Educ. 1990;54(11):680-687.

18. Dolan TA. The sensitivity of geriatric oral health assessment index to dental care. Journal of dental education. 1997;61(1):37-46.

19. Haikal DS. Oral health in a group of institutionalized elderly: selfperception, evaluation of observed conditions and impact on quality of life. $227 \mathrm{f}$ Dissertation (Master in Dentistry), School of Dentistry, Federal University of Minas Gerais, Belo Horizonte; 2004.

20. Bullinger M, Globe D, Wasserman J, et al. Challenges of patient-reported outcome assessment in hemophilia care - a state of the art review. Value Health. 2009;12(5):808-820.

21. Marx RG, Bombardier C, Hogg-Johnson S, et al. Clinimetric and psychometric strategies for development of a health measurement scale. $J$ Clin Epidemiol. 1999;52(2):105-111.

22. WHO (World Health Organization). WHOQOL and spirituality, religiousness and personal beliefs (SRPB) Field-Test Instrument, Switzerland. 1998. p. 1-25.

23. Almeida MAB , Gutierrez GL, Marques R. Quality of life: definition, concepts and interfaces with other areas of research -São Paulo: School of Arts. Science and Humanities -EACH/USP. p. 1-142.

24. Ikebe K, Hazeyama T, Enoki K, et al. Comparison of GOHAI and OHIP-14 measures in relation to objective values of oral function in elderly Japanese. Community Dentistry and Oral Epidemiology. 2012;40(5):406-414.

25. Vasconcelos LCA, Júnior RRP, Teles JBM, et al. Self-perceived oral health of the elderly in a medium-sized municipality in northeastern Brazil. Public Health Notebook. 2012;28(6):1101-1110.

26. Slade GD. Derivation and validation of a short-form oral health impact profile. Community Dent Oral Epidemiol. 1997;25(4):284-290.

27. Bombarba-Nunes FF, Miotto MHMB, Barcellos LA. Oral health selfperception of the community health agent of Vitória, ES, Brazil. Brazilian Research in Pediatric Dentistry and Integrated Clinic. 2008;8:7-14.

28. Coelho MP, Cordeiro MCP, Corrêa FF, et al. Assessment of the impact of oral conditions on quality of life measured by the OHIP instrument -14 . UFES Journal of Dentistry, Vitória. 2008;10(3):4-9.

29. Ferreira CA, Loureiro CA , Araújo VE. Psychometric properties of subjective indicator applied in children. Journal of Public Health. 2004;38(3):445-452.

30. Hassel AJ, Steuker B, Rolko C, et al. Oral health-related quality of life of elderly Germans: Comparison of GOHAI and OHIP-14 Community Dent Health. 2010;27(4):242-247.

31. Locker D, Matear D, Stephens M, et al. Comparison of the GOHAI and OHIP-14 as measures of the oral health-related quality of life of the elderly. Community Dent Oral Epidemiol. 2001;29(5):373-381. 
32. Locker D, Gibson B. Discrepancies between self-ratings of and satisfaction with oral health in two older adult populations. Community Dentistry and Oral Epidemiology. 2005;33(4):280-288.

33. Pandolfi M. Oral Health and Quality of Life of Users of Dental Services of Vitória (ES). Pes Bras Odontoped Clin Integr. 2011;11:311-316.

34. Montero J, López JF, Vicente MP, et al. Comparative validity of the OIDP and OHIP-14 in describing the impact of oral health on quality of life in a cross-sectional study performed in Spanish adults. Med Oral Patol Oral Cir Bucal. 2011;16(6):816-820.
35. Van Agt HM, Essink-Bot ML, Krabbe PF, et al. Test-retest reliability of health state valuations collected with the EuroQoL questionnaire. Soc Sci Med. 1994;39(11):1537-1544.

36. Kumar S, Bhargav P, Patel A, et al. Does dental anxiety influence oral health-related quality of life? Observations from a cross-sectional study among adults in Udaipur district, India. J Oral Sci. 2009;51(2):245-254. 